\title{
Superdisks and the structural asymmetry of radio galaxies
}

\author{
Gopal-Krishna ${ }^{a}$ Paul J. Wiita ${ }^{\mathrm{b}, *, 1}$ \\ ${ }^{a}$ National Centre for Radio Astrophysics, TIFR, Post Bag 3, Pune University \\ Campus, 41100\%, India \\ ${ }^{\mathrm{b}}$ Department of Physics and Astronomy, Georgia State University, Atlanta, GA, \\ 30302-4106, USA
}

\begin{abstract}
We present a sample of 16 radio galaxies, each of which is characterized by a wide, elongated emission gap with fairly sharp and straight edges between the two radio lobes. This particular subset of the "superdisk" radio galaxies is chosen because of a highly asymmetric location of the host elliptical galaxy relative to the gap's central axis. In addition to posing a considerable challenge to the existing models, such a morphology also means that the two jets traverse highly unequal distances through the superdisk material. One thus has a possibility to directly investigate if the marked asymmetry between the two jets' interaction with the (much denser) ambient medium, during their propagation, has a significant import for the brightness of the hotspot forming near each jet's extremity. We also propose a new explanation for the formation of superdisks through the merger of a smaller elliptical galaxy with the massive host, in which the gas attached to the infalling galaxy deposits its angular momentum into the host's circumgalactic gas, thereby causing it to flatten into a fat pancake, or superdisk. The asymmetric location of the host galaxy can be assisted by the kick imparted to it during the merger. We also suggest a physical link between these radio galaxies and those with $\mathbf{X}$-shaped and $\mathbf{Z}$-symmetric radio lobes, commonly believed to arise from mergers of two galactic nuclei, each harboring a supermassive black hole.
\end{abstract}

Key words: galaxies: active, galaxies: ISM, galaxies: jets, radio: continuum PACS: 98.54Ep, 98.54-w, 98.58.Fd, 98.65.Fz, 98.40.Dk

* Corresponding author.

Email addresses: krishna@ncra.tifr.res.in (Gopal-Krishna), wiita@chara.gsu.edu (Paul J. Wiita).

1 PJW acknowledges support from a sub-contract to GSU from NSF grant AST0507529 to the University of Washington. 


\section{Introduction}

A few years ago we noted that a fraction of extragalactic double radio sources exhibit radio morphologies and other properties that led us to infer that they required gaseous "superdisks" extending tens of kiloparsecs in both diameter and thickness (Gopal-Krishna \& Wiita, 2000, hereafter GKW00). The key morphological property shared by these radio galaxies is the sharp, quasi-linear edges of the radio lobes on the sides facing the central elliptical host galaxy (Gopal-Krishna \& Wiita, 1996; Gopal-Krishna \& Nath, 1997), as seen in at least a dozen radio galaxies at low to moderate redshifts. We showed that such superdisks (SDs) can provide consistent, alternative explanations for some key correlations found among the parameters of extragalactic radio sources, such as the Laing-Garrington effect (e.g., Garrington et al., 1988; Laing, 1988) and the correlated radio-optical asymmetries (e.g., McCarthy et al., 1991).

Furthermore, in high- $z$ RGs the strong tendency for redshifted component of the diffuse, quiescent Ly $\alpha$ emission to be on the side of the brighter hot spot has been explained by postulating that the redshifted Ly $\alpha$ emission originates from the region of the radio lobe on the near side of the nucleus, and is thus subject to much less dilution due to the intervening dust (e.g., Humphrey et al., 2007; Gopal-Krishna \& Wiita, 2005). As discussed in GKW00, these Ly $\alpha$ RGs provide optical evidence for high- $z$ SDs. Because the sharp-edged morphology would only be noticed when the radio jets are oriented close to the plane of the sky, SDs are subject to a strong negative selection effect, and GKW00 argued that even though the observed cases are few, the phenomenon may not be so uncommon. In this paper we present an enlarged sample of radio galaxies (RGs) exhibiting SDs and discuss, in particular, their properties related to structural asymmetry.

The sharp emission gaps seen in double radio sources are most commonly attributed to a blocking of the backflowing radio plasma in the lobes by a denser thermal plasma associated with the parent elliptical galaxy (e.g., Leahy \& Williams, 1984; Wiita \& Gopal-Krishna, 1990; Black et al., 1992). Such backflows are to be expected and simulations indicate that they will indeed be diverted by galactic ISM (e.g., Wiita \& Norman, 1992). This approach was an alternative to the original proposal by Scheuer (1974) according to which the central emission gap arose from the pinching of the inner parts of the lobes by the higher gas pressure of the host galaxy. Both of these mechanisms, however, seem incompatible with cases where the edges of the gaps are long and straight; they seem particularly incapable of explaining the highly asymmetric SDs where the host elliptical is found almost at one edge of the radio emission gap. A third possible explanation for large gaps has recently been put forward by Gergely \& Biermann (2007); they suggest that SDs could be carved out as two galaxy cores containing supermassive black holes (SMBHs) 
merge and the associated pair of jets undergoes a rapid precession during the later stages of the merger. While this novel process might indeed create a gap, it too may have difficulty in explaining the sharp inner edges of the lobes, particularly those of the highly asymmetric SDs we are exploring here.

Initially we proposed that the SD is primarily made of the interstellar medium bound to the RG itself, perhaps originating from the cool gas belonging to gasrich disk galaxies previously captured by the giant elliptical host of the powerful RG (e.g., Statler \& McNamara, 2002). We argued that the tidal stretching and heating of that gas during the capture was sufficient to produce very large fat pancakes (Gopal-Krishna \& Nath, 1997; Gopal-Krishna \& Wiita, 2000). Since in some extreme cases (e.g., 0114-476; Table 1) SDs were found to have widths running into several hundred kiloparsecs, an alternative possibility was also considered in GKW00, according to which at least some SDs trace the gaseous filaments of the "cosmic web".

In a recent paper, we have attempted to explain the occurrence of SDs in high$z$ RGs which have yet to acquire a significant circumgalactic medium (CGM) or reside in an intracluster medium (ICM) (ICM; Gopal-Krishna et al., 2007). We argued that the material forming the SD in such RGs likely arises from the nuclear wind which is believed to be associated with the AGN activity (e.g., Soker \& Pizzolato, 2005). We investigated the conditions under which this wind material would be squeezed between the two radio lobes into a pancake shaped SD. It was shown that for a wide range of reasonable wind and jet parameters those jets launched within a few tens of Myr subsequent to the initiation of wind production could quickly catch up to the wind-blown bubble and then the lobes growing outside the bubble could indeed squeeze the bubble in such a way as to produce SDs. Such a squeezing, however, is unlikely in $z<1$ RGs, which are usually surrounded by a significant amount of X-ray emitting CGM with a pressure at least that of the radio lobes (e.g., Croston et al., 2004). Therefore, another mechanism seems to be required at least for the SDs in low $z$ RGs.

In $\S 2$ we tabulate a significant number of additional RGs with highly asymmetric SDs. We also briefly discuss some interesting morphological properties of these asymmetric SDs. We then propose in $\S 3$ a new mechanism based on a merger of an elliptical galaxy, also rich in hot gas, with the massive host elliptical. In this scenario the orbital angular momentum of the captured galaxy's gas will be gradually transferred to the CGM gas of the host galaxy, thus transforming its quasi-spherical CGM halo into a fat pancake. This scenario ties in well with our explanation for the $\mathbf{Z}$-symmetries seen in some $\mathbf{X}$-shaped RGs (XRGs) (Gopal-Krishna et al., 2003, hereafter GKBW03). Conclusions are summarized in $\S 4$. 


\section{Radio Galaxies with Asymmetric Superdisks}

Previously we presented radio and optical evidences for SDs in a dozen powerful RGs (GKW00). In these objects the SDs are clearly traced by highly extended, sharp and quasi-linear inner edges of the radio lobes; these give rise to strip-like emission gaps between the lobe pair (also see Gopal-Krishna \& Wiita, 1996; Gopal-Krishna \& Nath, 1997). The median width of those SDs was found to be $\sim 25 \mathrm{kpc}$ and the median length at least three times greater.

One important RG correlation that is otherwise hard to explain is the lobedepolarization asymmetry (Laing, 1988; Garrington et al., 1988, 1991). The passage of radio photons through a very wide SD can naturally produce much greater depolarization for the more distant lobe, and this effect is practically independent of the extent of the RG (Gopal-Krishna \& Nath 1997). The previous explanation in terms of an essentially spherical magneto-ionic halo requires the unlikely condition that the size of that halo is always maintained to be within a factor of two of the total extent of the radio source (GKW00). The SD picture can also explain some meterwave flux variability via "superluminal refractive scintillations" (Gopal-Krishna, 1991; Campbell-Wilson \& Hunstead, 1994); also see Ferrara \& Perna (2001).

Another striking correlation exists between radio lobes and the extended optical emission line regions (EELRs), in the sense that the peak surface brightness of the EELR (on scales of tens of kpc) nearly always occurs on the side of the shorter of the two radio lobes (e.g., McCarthy et al., 1991). Their original explanation of this strong effect invoked large scale density asymmetries in the CGM about the RG; on the denser side, jet propagation would be slowed down and thermal emissivity of the EELR filaments enhanced through better confinement. Although conceivable, this mechanism cannot explain why the correlation should be nearly perfect (37 of 39 cases) even when the lobe size asymmetry is very minute. Nor can this McCarthy et al. (1991) interpretation explain why the brighter EELR - shorter lobe correlation is more pronounced for higher- $z$ RGs (where it is measured in the [O II] $\lambda 3727$ line) than for lower$z$ sources (measured in $\mathrm{H} \alpha$ ). An alternative explanation of this correlation in terms of SDs is discussed in Gopal-Krishna \& Nath (1997) and GKW00.

Our first sample of 12 SDs indicated that the hot spots in the radio lobe pair are usually located more symmetrically about the SD's midplane than about the host galaxy (GKW00). This greater symmetry with respect to the SD than relative to the current location of the host galaxy was clearly the case for 9 of the 12 RGs, and there was only one clear counterexample. The most plausible explanation for this result is that the galaxy has moved considerably from the SD's midplane since the AGN phase began. Such a motion would induce an asymmetry that would also work in the right direction to obscure the portion 
of the EELR which falls on the far side of the galaxy as marked by the current position of the radio core; furthermore, any dust in the SD would enhance the correlation in the blue (GKW00). Here we find that this trend for radio lobes to be more symmetric about the SD than about the radio nucleus is further strengthened in our enlarged sample of SDs.

In this paper we present an enlarged sample of 16 SDs, of which 6 are common to the set presented in GKW00. The selection criterion for the current sample is a pronounced asymmetry of the SD relative to the host elliptical galaxy, such that the galaxy is offset from the midplane of the SD by at least a quarter of the SD's average width; for this reason we do not include the other six RGs discussed in GKW00. While we do not claim the sample to be complete in any sense, we do expect it to be unbiased (and representative) from the viewpoint of the asymmetry, since it was assembled from a fairly extensive search of published radio maps such that no clear cases of asymmetric SDs were left out of this sample. Note that the source 3C 16 represents an extreme case of asymmetry, since the host galaxy lies completely outside the emission gap, within a radio lobe. The SD case is strong, and although the offset of the host from the center of the SD is large $(\sim 80 \mathrm{kpc})$, this distance could have been covered by the host galaxy moving at $\sim 350 \mathrm{~km} \mathrm{~s}^{-1}$ over an active lifetime of $\sim 250$ Myr (e.g., Barai \& Wiita, 2007). Another notable aspect of this sample (Table 1) is the presence of 3 double-double RGs (Lara et al.,, 1999; Schoenmakers et al., 2000), namely 3C 16, 0114-476 and 1155+266 (Table 1).

For each RG, identified in columns 1 and 2 of Table 1 , the redshift is given in column 3, while columns 4 and 5 give the largest angular size (LAS) of the RG and the width of the SD, respectively, in arcseconds. Column 6 converts that SD width to kpc, using a cosmology with $H_{0}=70 \mathrm{~km} \mathrm{~s}^{-1} \mathrm{Mpc}^{-1}, \Omega_{\Lambda}=0.7$ and $\Omega_{m}=0.3$. References to all of the radio maps are given in the last column of Table 1.

Contour maps (or radio photographs) of three of the RGs in our sample, namely, 3C 227, A1425 and 3C 381, are shown in Figs. 1-3, where the host galaxy is seen to be situated close to one edge of the SD. These maps are taken from Black et al. (1992), Owen \& Ledlow (1997) and Leahy \& Perley (1991), respectively.

In order to illustrate the way in which superdisks were identified and their properties measured, we show in Figs. 4-6 the outermost contour of the radio map of six of our SDs (see Table 1 for map references). In one case, 0114-476, a slightly higher contour is reproduced in order to illustrate the outline of the SD more clearly. The ' + ' sign marks the radio core at the nucleus of the host galaxy. Also reproduced are one or two contours closely encircling the peak of the hot spot within each radio lobe. In the case of 0114-476, the inner double lobes are also detected and are shown. The edges of each SD, as adopted here, 


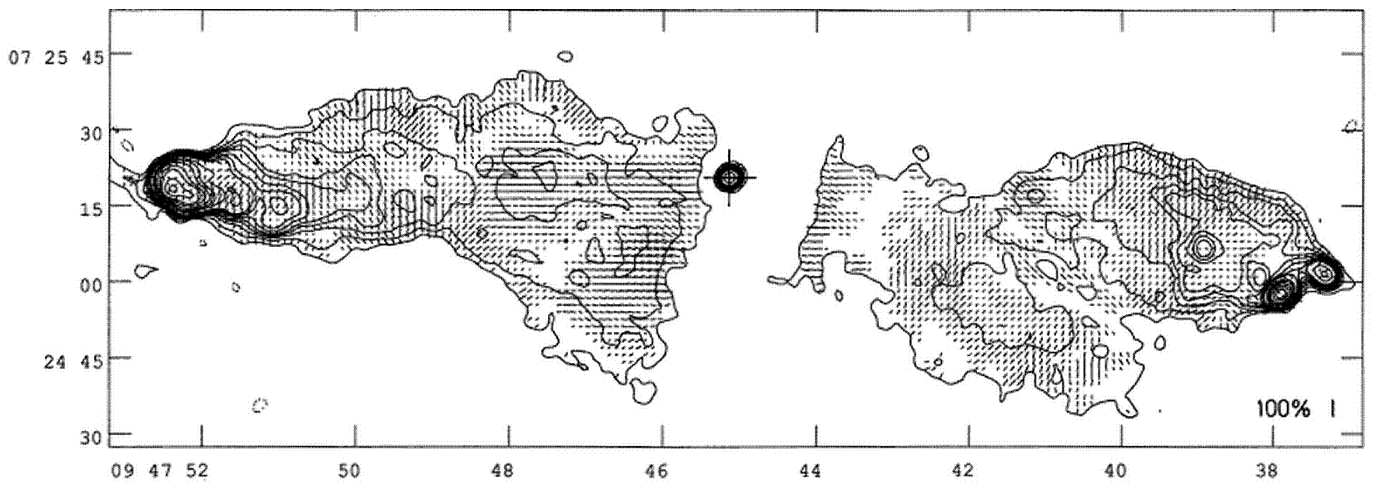

Fig. 1. Radio map of 3C $227(0945+076)$ from Black et al. (1992). Contours are shown at $(-3,3,6,9,12,15,20,25,30,50,70,100,150,200,300,400,500) \times 150$ $\mu \mathrm{Jy}$ beam $^{-1}$. Percentage polarization values (E vectors) are also shown.C Royal Astronomical Society; reproduced by permission of the RAS.

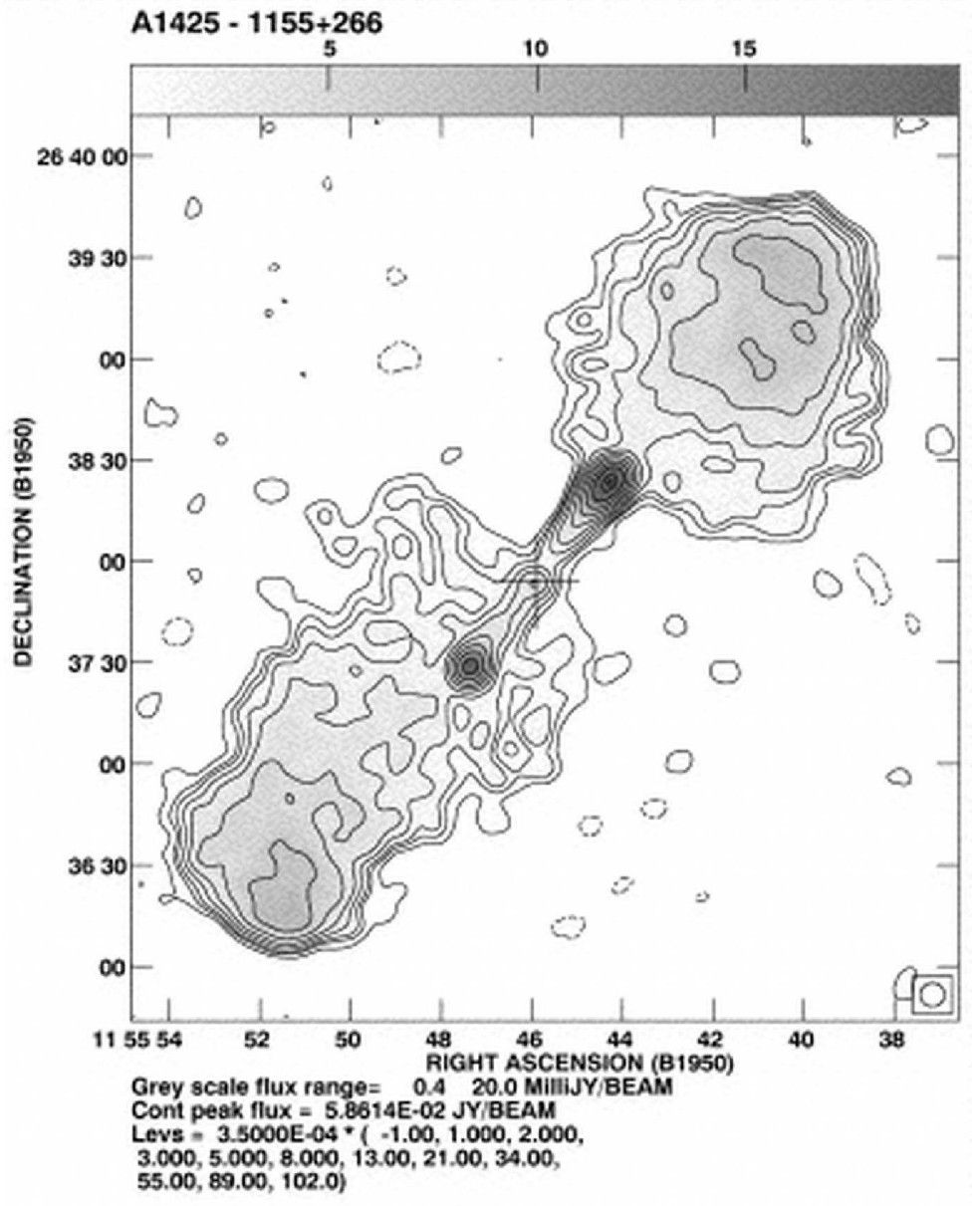

Fig. 2. Radio map of A1425 (1155+266) from Owen \& Ledlow (1997); (C)American Astronomical Society; reproduced by permission of the AAS. 


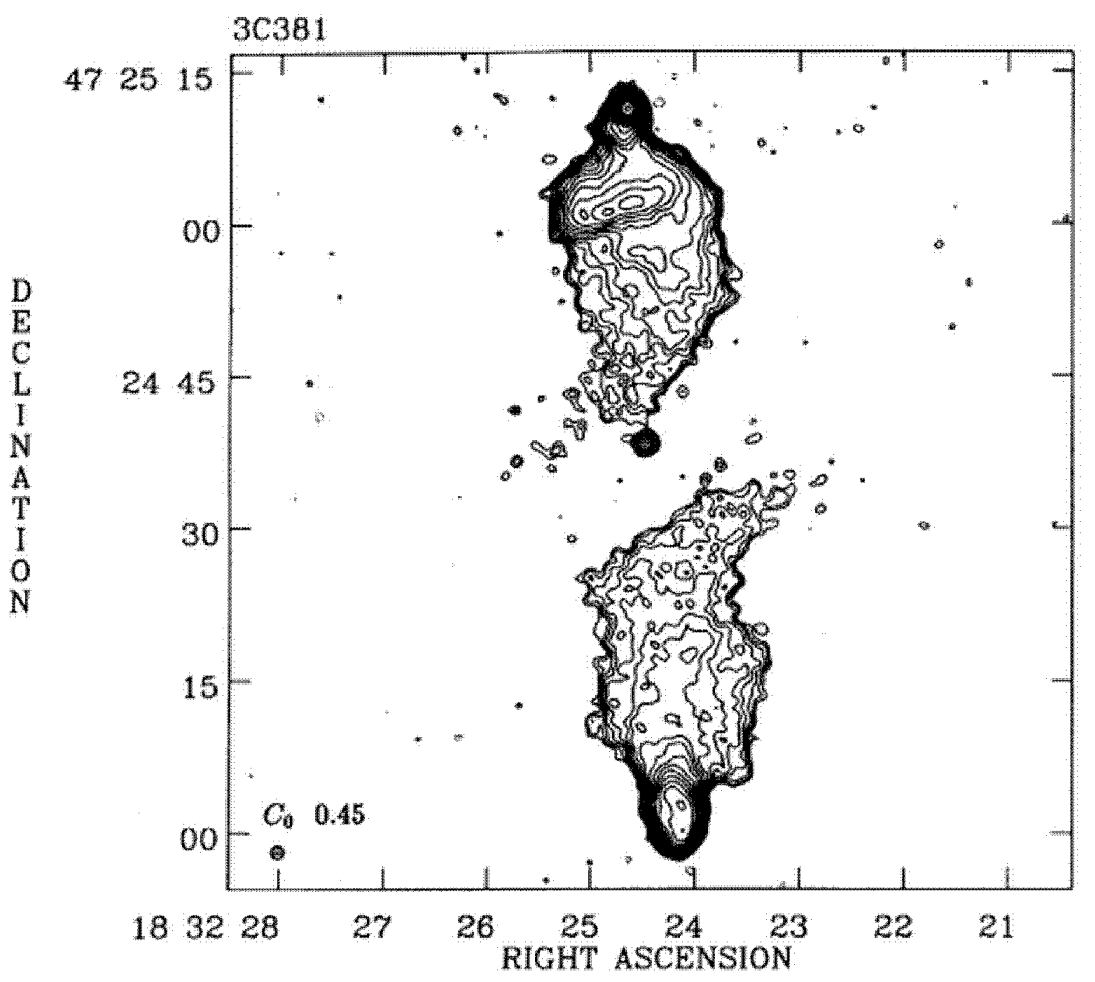

Fig. 3. Radio map of 3C $381(1832+474)$ from Leahy \& Perley (1991). Contours are logarithmic with a ratio of $\sqrt{2}$ between levels; i.e., levels are at $\left.C_{0} \times 1, \sqrt{2}, 2,2 \sqrt{2}, 4, \ldots\right)$. The value of $C_{0}=0.45 \mathrm{mJy}$ beam $^{-1}$ is given in the panel and is about 3 times the rms noise level; the hatched circle indicates the FWHM restoring beam. (C)American Astronomical Society; reproduced by permission of the AAS.

are bracketed by a pair of straight lines and the central axis (i.e., mid-plane) of the SD is indicated with a broken line. In all cases, the published radio contours allow us to delineate fairly well the two edges of the SDs, despite relatively narrow protrusions into the SD region which are seen in some of the RGs and are probably associated with the radio jets.

We first summarize some salient features of the present sample of 16 RGs. They lie within a redshift range from $\sim 0.04$ to $\sim 0.6$ and are thus in the cosmic age regime where each should have acquired a substantial amount of hot circumgalactic gas. In all cases the optical counterpart is a galaxy; this is expected since the sharp-edged gaps in the radio bridge are likely to be observed only when the jets are close to the sky plane (a necessary condition for identification as a galaxy, and not a quasar, according to the unified scheme for radio powerful AGN, e.g., Barthel, 1989). Another property consistent with the unified scheme is that kpc-scale jets are clearly visible in just a few of these RGs, since Doppler boosting will not be significant for the jet emission.

Projected linear sizes of these 16 RGs range between 28 and $1620 \mathrm{kpc}$ while the widths of the central emission gaps are between $\sim 8$ and $510 \mathrm{kpc}$. Discarding 

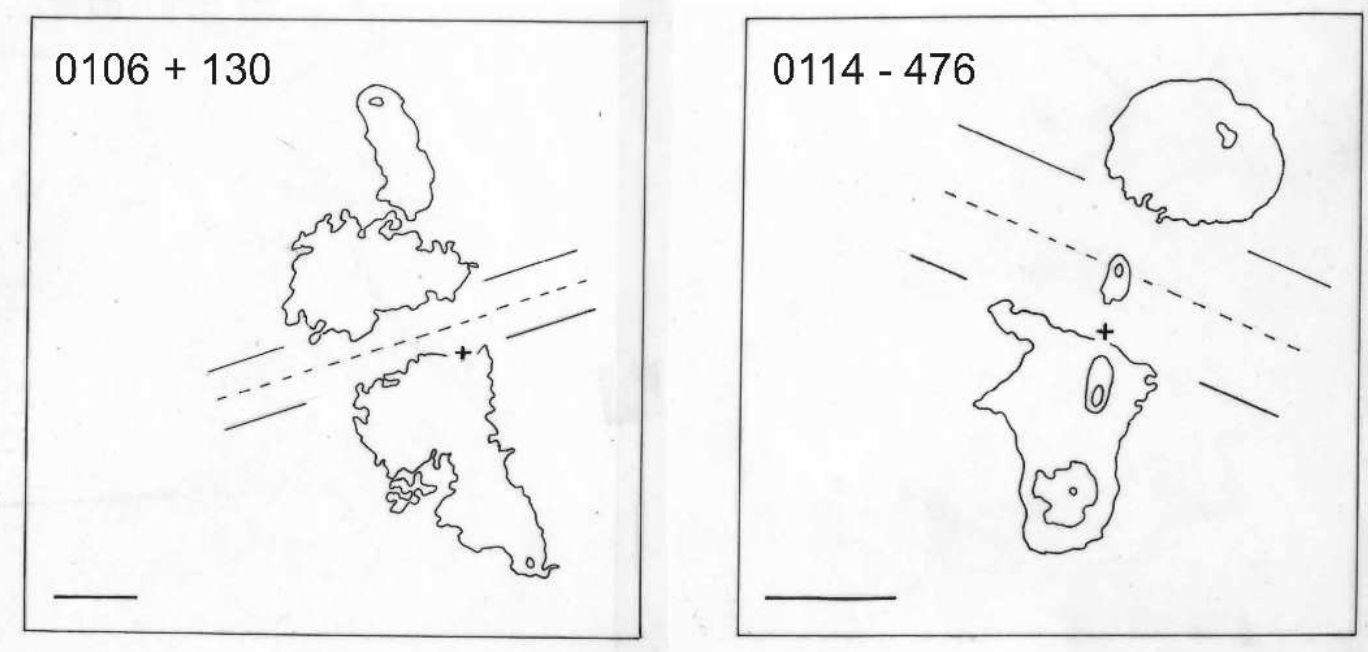

Fig. 4. Outlines of radio maps of $0106+130$, 0114-476, with ' + ' signs giving the location of center of the host galaxies, straight line segments illustrating the edges we adopt for the SD and dashed lines giving the SD midplanes. The hotspot location is noted for each lobe and $50 \mathrm{kpc}$ scale bars are drawn in each panel, except for the RG 0114-476, where it measures $500 \mathrm{kpc}$. The maps from which these contours were traced are respectively from references 2 and 5 in Table 1.
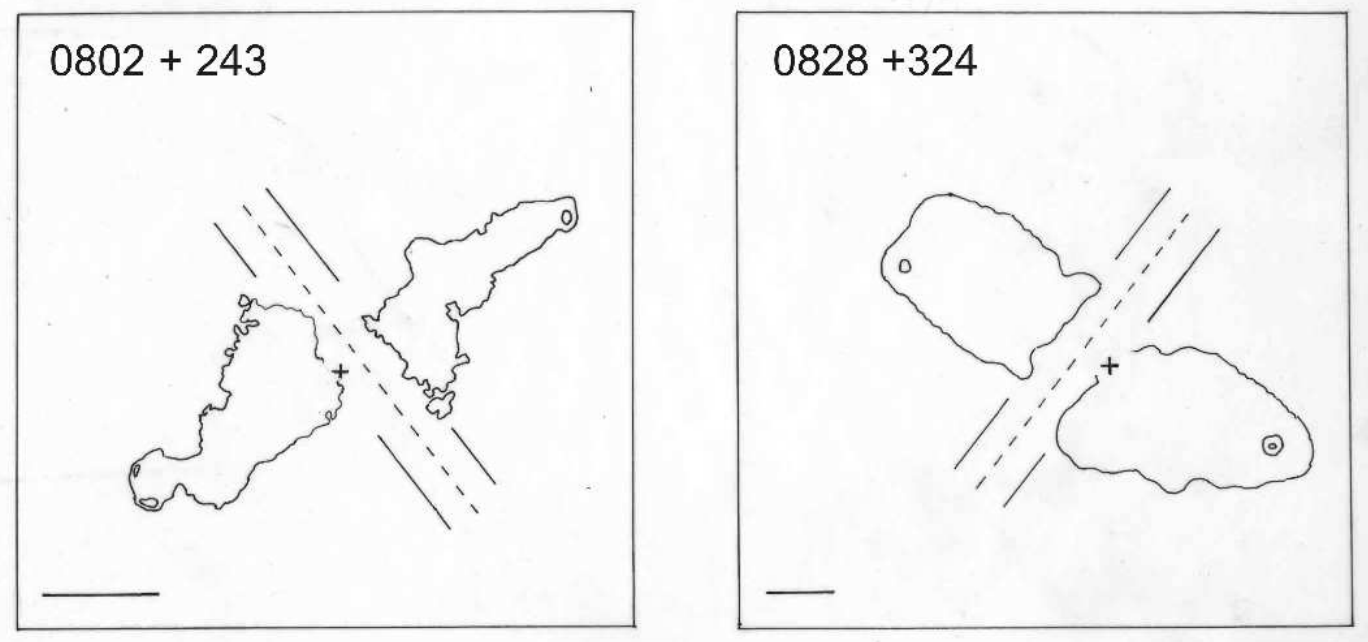

Fig. 5. As in Fig. 4 for the RGs 0802+243, 0828+324, from references 9 and 11.

these two extreme values, the mean of the other 14 projected SD widths is 37 $\mathrm{kpc}$ and the median is $31 \mathrm{kpc}$. Nuclear radio cores are detected in 12 of the 16 RGs (see references in Table 1).

Column 7 of Table 1 identifies the brighter hot spot and also gives $R$, the ratio of the highest contour values for the two hotspots in the opposite lobes. Column 8 gives the ratios, $Q$, of the distances of those two hot spots from the radio core (or host galaxy). The ratios, $Q_{\mathrm{sd}}$, of the distances of the hot spots from the mid-plane of the SD, as measured from the intersection of 

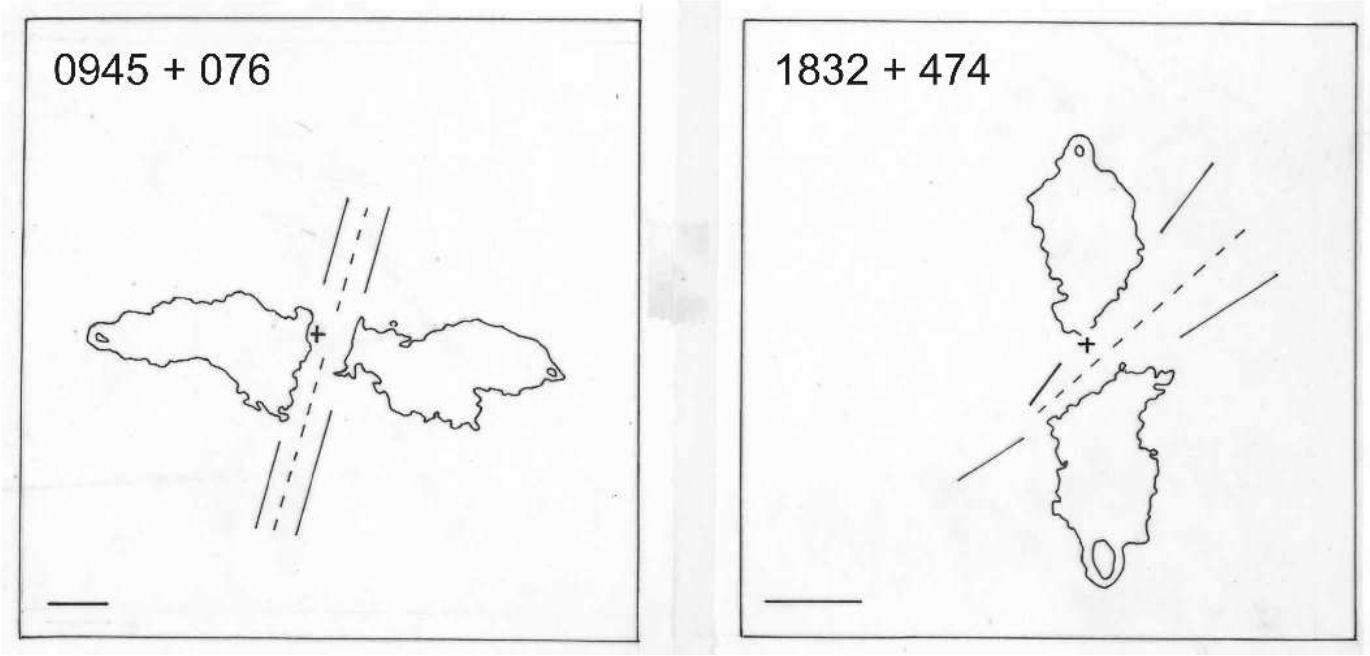

Fig. 6. As in Fig. 4 for the RGs 0945+076, and 1832+474, from references 12 and 2 .

the radio axis (i.e., the line joining the two hot spots), are given in Column 9. Here we define $Q$ and $Q_{\text {sd }}$ so that they correspond to the distance to the brighter hotspot divided by the distance to the fainter hot spot. The selection of the brighter of the two hot spots is done by comparing the highest contour values read from the radio maps cited in Table 1 and partly reproduced in the captions to Figs. 1-3. Defined in this manner, and based upon multiple measurements by both authors, the standard errors on $Q$ are $\sim 0.01$; however, because of the uncertainty in the exact location of the midplane of the SD, the errors on $Q_{s d}$ are a bit larger, but still $\lesssim 0.03$. Column 10 ("SD Sym") indicates whether the value of $Q_{\text {sd }}$ (or its inverse) is closer to 1 than is $Q$, i.e., if the lobe extents are more symmetrical with respect to the superdisk's midplane than relative to the nucleus. Column 11 gives the misalignment angle, $\psi$, defined as the supplement to the angle between the lines connecting the two hotspots to the nucleus. In column 12 ("Fade") ' $\mathrm{Y}$ ' indicates when the jet traversing the greater distance through the SD produces the fainter of the two hotspots.

Fig. 7 gives a histogram of values of $Q_{s d}$ and $Q$ where we discard the hot spot brightness ratio criterion and so define each value such that $Q \geq 1$; i.e., if the value in Table 1 is $<1$ we replace it by its inverse. As noted for a smaller sample in GKW00, the values of $Q_{\text {sd }}$ are usually closer to 1 than are those of $Q$. The present larger sample strengthens this trend; the probability of having 12 (or greater) Y's in Column 10 by chance is only 0.038 . This highly significant correlation is consistent with the scenario that at the time of jet launching the RGs were fairly centrally situated within their respective SDs and have gradually drifted outward since then (see GKW00).

Another suggestive correlation is noted for the cases where the sense of $Q$ and $Q_{\text {sd }}$ are found to be opposite, with one ratio, nearly always $Q$, less than one, while $Q_{\text {sd }}>1$. In every case where $\psi \geq 14^{\circ}$, i.e., 3C 16, 0114-476, 3C 401 
Table 1

Radio Galaxies with Asymmetric Superdisks

\begin{tabular}{llccccccccccc}
\hline Source & Other & $\mathrm{Z}$ & $\mathrm{LAS}$ & $\mathrm{SD}$ & $\mathrm{Width}$ & $\mathrm{R}^{a}$ & $\mathrm{Q}$ & $\mathrm{Q}_{\text {sd }}$ & $\mathrm{SD} \mathrm{Sym}$ & $\psi$ & Fade & Refs \\
& Name & & $\prime \prime$ & $\prime \prime$ & $\mathrm{kpc}$ & & & & & $\circ$ & & \\
\hline $0035+130^{b}$ & $3 \mathrm{C} 16$ & 0.405 & 73 & 13 & 70 & $\mathrm{~S} 32$ & 0.49 & 1.82 & $\mathrm{Y}$ & 14 & $\mathrm{Y}$ & 1,2 \\
$0106+130^{b}$ & $3 \mathrm{C} 33$ & 0.060 & 70 & 8 & 9 & $\mathrm{~S} 5.7$ & 0.82 & 1.08 & $\mathrm{Y}$ & 2 & $\mathrm{Y}$ & 2 \\
$0106+729$ & $3 \mathrm{C} 33.1$ & 0.181 & 55 & 19 & 58 & $\mathrm{~N} 6.4$ & 0.59 & 0.96 & $\mathrm{Y}$ & 0 & $\mathrm{Y}$ & 3,4 \\
$0114-476$ & & 0.146 & 635 & 200 & 510 & 1 & 0.71 & 1.34 & $\mathrm{Y}$ & 20 & $?$ & 5 \\
$0117-156$ & $3 \mathrm{C} 38$ & 0.565 & 13 & 3 & 19 & $\mathrm{~N} 2$ & 0.52 & 0.95 & $\mathrm{Y}$ & 11 & $\mathrm{Y}$ & 6 \\
$0453+227$ & $3 \mathrm{C} 132$ & 0.214 & 22 & 4 & 14 & $\mathrm{~W} 2$ & 1.06 & 1.23 & $\mathrm{~N}$ & 2 & $\mathrm{Y}$ & 7 \\
$0528+064$ & $3 \mathrm{C} 142.1$ & 0.406 & 49 & 6 & 33 & $\mathrm{~W} 2$ & 0.61 & 0.52 & $\mathrm{~N}$ & 10 & $\mathrm{~N}$ & 8 \\
$0605+480$ & $3 \mathrm{C} 153$ & 0.277 & 7 & 2 & 8 & $\mathrm{E} 2$ & 1.95 & 2.52 & $\mathrm{~N}$ & 1 & $\mathrm{~N}$ & 7 \\
$0802+243^{b}$ & $3 \mathrm{C} 192$ & 0.060 & 190 & 27 & 31 & $\mathrm{~W} 4$ & 1.18 & 1.02 & $\mathrm{Y}$ & 5 & $\mathrm{~N}$ & 9,10 \\
$0828+324^{b}$ & $4 \mathrm{C} 32.25$ & 0.051 & 307 & 52 & 52 & $\mathrm{~W} 2.9$ & 0.80 & 1.09 & $\mathrm{Y}$ & 0 & $\mathrm{Y}$ & 11 \\
$0945+076^{b}$ & $3 \mathrm{C} 227$ & 0.086 & 230 & 23 & 37 & $\mathrm{E} 2$ & 1.08 & 1.01 & $\mathrm{Y}$ & 10 & $\mathrm{Y}$ & 12 \\
$1155+266$ & $\mathrm{~A} 1425$ & 0.140 & 240 & 50 & 123 & 1 & 0.92 & 1.46 & $\mathrm{~N}$ & 1 & $?$ & 13 \\
$1409+524$ & $3 \mathrm{C} 295$ & 0.464 & 4.7 & 1.7 & 10 & $\mathrm{~N} 1.8$ & 0.67 & 1.12 & $\mathrm{Y}$ & 0 & $\mathrm{Y}$ & 14 \\
$1832+474^{b}$ & $3 \mathrm{C} 381$ & 0.161 & 71 & 11 & 30 & $\mathrm{~N} 9$ & 0.86 & 1.05 & $\mathrm{Y}$ & 1 & $\mathrm{Y}$ & 2 \\
$1939+605$ & $3 \mathrm{C} 401$ & 0.201 & 22 & 3 & 10 & 1 & 0.70 & 1.10 & $\mathrm{Y}$ & 15 & $?$ & 7,15 \\
$2104-256$ & $\mathrm{NGC} 7018$ & 0.039 & 112 & 35 & 27 & $\mathrm{~N} 1.4$ & 0.67 & 1.35 & $\mathrm{Y}$ & 18 & $\mathrm{Y}$ & 16 \\
\hline
\end{tabular}

Notes: ${ }^{a}$ the brighter hotspot (E or $\mathrm{W} ; \mathrm{N}$ or $\mathrm{S}$ ) and the ratio of its peak contour to the fainter's; ${ }^{b}$ also in Table 2 of GKW00. References: 1) Harvanek \& Hardcastle (1998), 2) Leahy \& Perley (1991), 3) van Breugel \& Jagers (1982), 4) DRAGN atlas: www.jb.man.ac.uk/atlas, 5) Saripall et al. (2002), 6) Morganti et al. (1999), 7) Hardcastle et al. (1997), 8) Bogers et al. (1994), 9) Leahv et al. (1997), 10) Baum et al. (1988), 11) Capetti et al. (1993), 12) Black et al. (1992), 13) Owen \& Ledlow (1997), 14) Perley \& Tavlor (1991), 15) Burns et al. (1984), 16) Morganti et al. (1993)

and NGC 7018, this ratio is inverted, though such a trend is also found for a few cases with smaller $\psi$ (i.e., 3C 33, 4C32.25, and 3C 381, Table 1). Thus, while not a strong correlation, the tendency of the double radio sources with highly misaligned hotspots (i.e., larger $\psi$ ) to also have clearly flipped values of $Q$ and $Q_{\text {sd }}$ is consistent with the notion of the host galaxy moving relative to the SD, as inferred above.

A stronger correlation found in the present sample is for the fainter of the 


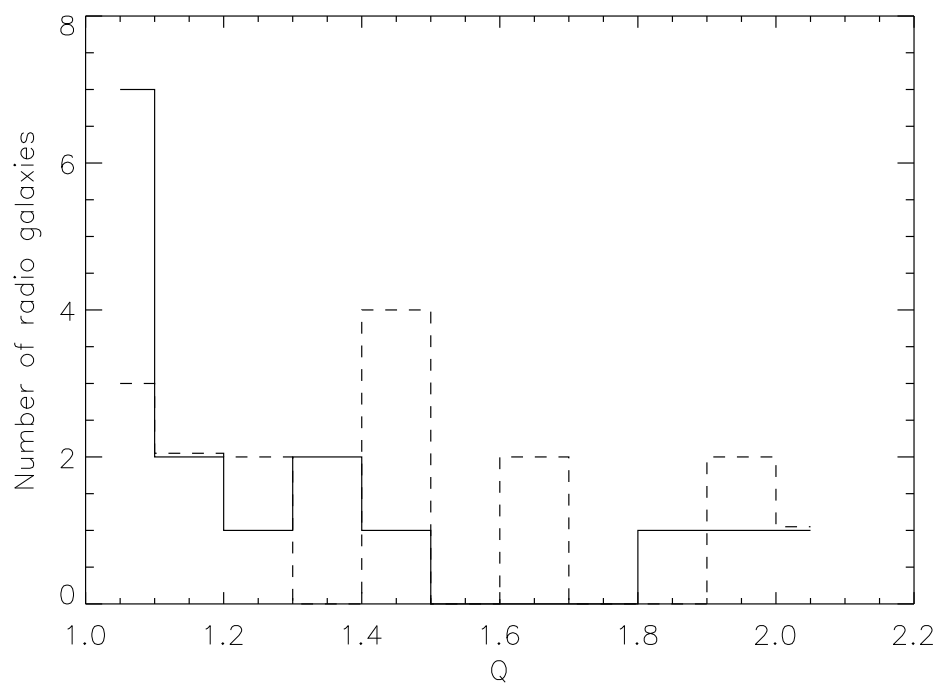

Fig. 7. Histograms of the distributions of $Q_{\text {sd }}$ (solid lines) and $Q$ (dashed lines); the last bin is comprised of $Q_{\mathrm{sd}}=2.50$ along with $Q=2.04$.

two hotspots to be found at the termination of the jet that travels a greater distances through the SD. In 13 of the 16 RGs, the brighter of the two hot spots can be unambiguously identified. Two of the three RGs where the fluxes are nearly equally bright, 0114-476 and A1425, are double-double sources, where the weakened outer hot spots are probably not being currently fed (Schoenmakers et al., 2000). Of these 13 sources with clearly unequal hotspot fluxes, 10 show a fainter hotspot to be associated with jet traversing a longer column of the SD material (column 12 of Table 1). The probability of 10 or more out of 13 cases adhering to this trend is only 0.046 . Such a behavior can be explained by a stronger interaction of the jet traversing the denser SD material, conceivably causing a larger entrainment of that material into the jet, as compared to the jet propagating mostly through the much lighter lobe plasma.

\section{Rotating the Circumgalactic Medium}

To produce a powerful RG a massive galaxy is required (e.g., Jahnke \& Wisotzki, 2003) and therefore at least one significant merger (defined as a merger one where the ratio of masses exceeds 0.1 ) is to be expected in the life of that galaxy (e.g., Parkinson et al., 2008). Such a merger, via spinning up the resultant black hole, may trigger the jet formation (e.g., Wilson \& Colbert, 1995), although it is now becoming clear that not all powerful RGs show clear signs of major mergers, i.e., those where the galaxy mass ratio exceeds 0.3 (Tadhunter et al., 2007), and so such mergers are not required for incite- 
ment of RG activity. The usual environment for powerful RGs is a group or sparsely populated cluster of galaxies (e.g., Wold et al., 2000). To check the feasibility of the angular momentum transfer concept put forward in Sect. 1, we carry out a rough estimation using reasonable fiducial values for the various parameters. So we consider a massive elliptical host galaxy, of baryonic mass $M_{h} \simeq 5 \times 10^{11} M_{\odot}$, capturing a smaller, but still significant, elliptical group member, of mass $M_{s} \gtrsim 1 \times 10^{11} M_{\odot}$. This galaxy is taken to initially have a mass, $M_{g}=f M_{s}$, of hot gas, with $f \lesssim 0.1$. The larger galaxy will possess a hot halo of gas, which we shall denote as its CGM, with typical core radius, $a \approx 1 \mathrm{kpc}$, central density $n_{0} \approx 10^{-1} \mathrm{~cm}^{-3}$, and density distribution $n_{C G M}(r) \approx n_{0} /\left[1+(r / a)^{2}\right]^{-\beta}$, with $\beta \simeq 0.6$ and temperature, $T \simeq 10^{7} \mathrm{~K}$ (e.g., Mathews \& Brighenti, 2003).

As the smaller galaxy moves toward the host, with a typical initial speed of $V_{s} \simeq 300 \mathrm{~km} \mathrm{~s}^{-1}$, (appropriate for a group with a $1 \mathrm{keV} \mathrm{X}$-ray temperature; e.g., Jeltema et al., 2006), its gas will be subject to ram pressure stripping by the CGM. However, only the outer portions of this gas are likely to be directly stripped at large distances from the host where $n_{C G M}<10^{-3} \mathrm{~cm}^{-3}$, in that $M_{s}=1.0 \times 10^{11} M_{\odot} \gg M_{I S}$, where the mass below which the simple ram pressure stripping is efficient is given by (Mori \& Burkert, 2000),

$$
\begin{aligned}
M_{I S}=1.27 \times 10^{9} & (f / 0.1)^{-7 / 2}\left(n_{C G M} / 10^{-4} \mathrm{~cm}^{-3}\right)^{7 / 2} \\
& \times\left(V_{s} / 10^{3} \mathrm{~km} \mathrm{~s}^{-1}\right)^{7} \mathrm{M}_{\odot}
\end{aligned}
$$

A second phase of slower stripping, basically due to the Kelvin-Helmholtz instability, still will occur, but simulations show that its timescale should exceed $2 \times 10^{9}$ yr for these parameters (Mori \& Burkert, 2000; Roediger \& Hensler, 2005). By that time the merger should be complete if the smaller galaxy is fairly compact and its impact parameter, $b \lesssim R_{c}$, where $R_{c} \simeq 10 \mathrm{kpc}$ is the galactic half-light radius (e.g., Bertin et al., 2003; González-García \& van Albada, 2005).

Therefore, at the point of the approaching galaxy's entry into the inner part of the CGM a significant fraction $\eta$, of the infalling galaxy's gas is taken to be retained. The initial angular momentum of the gas associated with the galaxy to be captured is $\ell_{i}=\eta M_{g} b V_{s}$. The gravitational interaction between the stars of the two galaxies leads to the merger through dynamical friction and tidal stripping; then the separation between the remainder of the infalling galaxy and the distorted host (e.g., González-García \& van Albada, 2005) will be $r_{f}<R_{c} / 2$. Any remaining orbital velocity must be less than the velocity dispersion of the host, so $V_{f} \lesssim 200 \mathrm{~km} \mathrm{~s}^{-1}$. Therefore the ISM core of the infalling galaxy will also have shared the bulk of its angular momentum with the larger galaxy's gas. The decrease in the angular momentum of the infalling 
galaxy's gas will be

$$
\Delta \ell=\ell_{i}\left[1-\left(r_{f} / b\right)\left(V_{f} / V_{s}\right)\right]
$$

Given the impact parameter required for the merger to occur, the bulk of this angular momentum must be taken up by the gas of the inner portion of the CGM, which will acquire a global orbital speed,

$$
\left\langle V_{C G M}\right\rangle=\Delta \ell / M_{C G M}\langle R\rangle,
$$

where the angle brackets denote mass weighted averages. For example, with $R_{\max , C G M}=20 \mathrm{kpc}, M_{C G M} \simeq 2.3 \times 10^{9} M_{\odot}$ and $\langle R\rangle \simeq 12 \mathrm{kpc}$. Then, for $\eta=0.8$ and $f=0.1$ we find from Eq. (3) that $\left\langle V_{C G M}\right\rangle \simeq 580 \mathrm{~km} \mathrm{~s}^{-1}$. Such a very large orbital velocity for the gas (and not for the stars) means that enough angular mometum has been injected into the gas to transform the initially quasi-spherical CGM halo, with a thermal (sound) speed, $V_{t} \simeq 470$ $\mathrm{km} \mathrm{s}^{-1}$, into a fat pancake of radius approaching $40 \mathrm{kpc}$. This expansion would take a time $\tau \gtrsim 40 \mathrm{kpc} / \mathrm{V}_{\mathrm{t}} \simeq 8 \times 10^{7} \mathrm{yr}$. As this is much less than the time for the merger to be completed, we see that this merger mechanism does provide a viable route to the formation of a wide, flattened pancake of gas. Recently, Di Matteo et al. (2007) have performed an extensive suite of simulations of mergers and fly-bys of elliptical-elliptical, elliptical-spiral and spiral-spiral pairs, including both co- and counter-rotating interactions at various peri-centers. The focus of these simulations was on the amount and location of the enhancement of star formation, and they assume that the elliptical galaxies are devoid of the relatively cool $\left(\sim 10^{4} \mathrm{~K}\right)$ gas that can plausibly produce stars, while they ignore the hot gas $\left(\sim 10^{7} \mathrm{~K}\right)$ known to be present around ellipticals and which we utilize in the above calculations. Di Matteo et al. (2007) show that the results depend sensitively on the orientations of disks and on the maximum strength of the tidal interactions; while their simulations are not directly relevant to the situation we are treating, they do clearly show that in some cases, large fractions of the gas can be ejected far from the center of the merged galaxies, in essential agreement with our argument earlier in this paragraph. In our rough estimate we have not explicitly considered the ram pressure stripping that removes more of the gas from the infalling galaxy as it traverses the denser innermost portions of the CGM. However, the angular momentum of this gas will also be absorbed by the CGM, so this should not drastically affect our argument. Our calculation is merely a demonstration that for reasonable parameter values, not quite major mergers of elliptical galaxies can yield very expanded hot gaseous SDs and we expect (and require) that they will actually form in only a minority of all elliptical mergers.

Observational evidence for rotation in the gas surrounding RGs comes from 
optical $(\mathrm{H} \alpha)$ spectroscopy (Noel-Storr et al., 2003) although the galaxies in this sample are weaker, nearby $(z<0.023)$, FR I RGs. Unfortunately, the number of FR II RGs at redshifts low enough to allow measurements that could clearly detect the rotation of the hot associated gas is small, so direct evidence for rotation in the type of $\mathrm{RG}$ we are considering is not yet available. Additional indirect evidence for large-scale rotation in the CGM comes from the comparison of observed and simulated radio source morphologies in nearby clusters of galaxies (Heinz et al., 2006). Detailed simulations of merging galaxies in massive, high- $z$ halos suggest that large quantities of molecular gas form and gradually evolve into a rotational configuration and eventually into a disky structure, in agreement with sensitive CO line observations of quasars (Narayanan et al., 2006; Carilli \& Wang, 2006).

One caveat to our merger scenario is the possibility that the gas stripped from the smaller galaxy will, to some degree, drive turbulence which might dissipate some of the angular momentum before it can be widely shared by the CGM. Simulations indicate that this effect is probably minor under most circumstances (e.g., Roediger \& Hensler, 2005), but no extant simulations of which we are aware are directly applicable to the situation envisioned here. It is worth noting that the presence of even weak magnetic fields in the CGM will substantially help stabilize the relatively flat boundary between the SD and the radio lobes against surface instabilities (e.g., simulations by Jones \& De Young, 2005). The stabilizing role of this magnetic tension is may well be indicated by the commonly observed good alignment of the magnetic field within the radio lobes along their quasi-linear inner edges (Fig. 1; Table 1).

The final stage of the galactic merger, particularly once the two SMBHs merge, is very likely to trigger activity in the nucleus. We earlier considered (GKBW03) how a rotating stream of the CGM gas could explain the $\mathbf{Z}$-symmetry detected in a few well mapped $\mathbf{X}$-shaped RGs (XRGs). The currently favored mechanism for XRGs involves the reorientation of the spin axis of the SMBH in the dominant galaxy following merger with another substantial BH at the core of the captured galaxy (e.g., Rottmann, 2001; Zier \& Biermann, 2002; Merritt \& Ekers, 2002). Before the merger, a pair of jets is feeding one pair of radio lobes, but the merger temporarily disrupts the accretion disk, and thus the jets. The post-merger SMBH has its spin axis reoriented, largely at the expense of the orbital angular momentum of the captured BH (e.g., Merritt \& Ekers, 2002; Zier \& Biermann, 2002). It then can reestablish new jet flows along this flipped axis. This type of rapid reorientation of the RGs jets can nicely explain the morphological and spectral properties of the XRGs. Some of these new jet pairs should naturally develop a $\mathbf{Z}$-symmetry while traversing the CGM already set in rotation if, as is expected, the CGM and reoriented SMBH do not have a common rotation axis (Gopal-Krishna et al., 2003; Zier, 2005). 
In a small fraction of SMBH mergers, however, no spin-flip will occur because the orbital axis of the infalling BH happens to be close to the original spin axis of the central engine. For such cases the axis of the flattened CGM disk will also be close to both the old and new jet axes; therefore, the jets will continue to propagate undeflected. Such undeflected "naked jets" are visible, for example, in the radio sources 3C 28 and 3C 153 (DRAGN atlas: www.jb.man.ac.uk/atlas). The blocking of the backflow by the thick and now flattened pancake of CGM would give rise to the strip-like emission gap between the radio lobes, i.e., the SD. While the rotation of the CGM disk would have no major influence on the propagation of the stiff jets, the lobe plasma within the region of the rotating CGM is likely to be peeled off of the jets and mixed into the CGM, thereby causing a drastic fading of the lobe radio emission within the disk. Such residual radio emission within the SD could sometimes still be detected; some examples are the RGs J1137+613 (Lara et al., 2001) and 3C 192, where a deeper map at lower frequency shows faint radio emission coinciding with the SD (Baum et al., 1988).

Note that in our scenario, the number of SD sources should be significantly smaller than that of the XRGs, as they both arise from SMBH mergers, but the former requires a preferred orientation. Until recently, however, RGs showing SDs and $\mathbf{X}$-shaped lobes were known in comparable numbers, thereby posing an apparent inconsistency. However, a recent analysis of the FIRST (Becker et al., 1995) survey has led to the discovery of over 100 new XRG candidates (Cheung, 2007), so the relative numbers of XRGs and SDs no longer seems to be inconsistent with our proposal.

To understand the asymmetrical placement of the host galaxy with respect to the center of the emission gap in the sources we have focused on in this paper, we require the presence of a modest component of the motion of the host galaxy along the direction of the jets (GKW00). This would be $\sim 100$ $\mathrm{km} \mathrm{s}^{-1}$ to cover the typical offset distance of $\sim 10 \mathrm{kpc}$ in a RG lifetime of $\sim 10^{8}$ yr (e.g., Barai \& Wiita, 2007). Some additional movement of the host, roughly along the radio axis, arises from the linear momentum imparted by the incoming galaxy at the time of merging. However, for the mass ratio (1:5) we have taken as fiducial, and for a component of the galaxy's velocity along the axial direction of $V_{k}=3^{-1 / 2} V_{s} \simeq 170 \mathrm{~km} \mathrm{~s}^{-1}$, this kick amounts to only $\sim 30$ $\mathrm{km} \mathrm{s}^{-1}$. The merger is therefore unlikely to cause the bulk of the asymmetry, which must be provided by random motion of the host galaxy; however, it certainly can contribute to it.

In any event, the present finding that the lobe pair is more symmetric relative to the SD than to the host galaxy distinctly favors the scenario in which motion of the galaxy relative to the SD material, during the lifespan of radio activity, makes a substantial contribution to the observed lobe-length asymmetry measured relative to the radio core (GKW00). Here it is worth reiterating that 
although orientation effects can significantly distort the observed properties of most samples of RGs (e.g., Humphrey et al., 2007; Gopal-Krishna \& Wiita, 2005), they are expected to be minimal for RGs exhibiting signatures of superdisks (Sect. 1). An examination of asymmetry in the kinematics of quiescent gaseous halos associated with powerful RGs and their radio parameters, such as the brightness of jets, hotspots and the spectral index, led Humphrey et al. (2007) to conclude that while the statistically significant correlations found among these parameters can be reasonably explained in terms of orientation effects, the predicted pattern of radio lobe-length asymmetry is, however, not observed. They attribute this apparent discrepancy to the primary influence of environmental asymmetry on the lobe length attained. In our picture, this decoupling of the lobe-length asymmetry from the various orientation induced correlations can be understood in terms of a large contribution to the lobe-length asymmetry coming from the galaxy motion (which is orientation independent), as mentioned above.

\section{Conclusions}

We have highlighted a particularly interesting and intriguing class of RGs which not only shows an SD type morphology but also the host galaxy is seen close to the edge of the SD and is thus grossly offset from the midplane of the SD. This morphological asymmetry poses a serious difficulty for existing SD models, particularly when the superdisk is found in RGs at $z \lesssim 1$, where a significant amount of hot gas surrounding the host is usually present. We have shown that the two radio lobes extend significantly more symmetrically about the SD midplane than they extend relative to the host galaxy.

We have proposed a scenario whereby gaseous SDs acquire their form through the injection of angular momentum from the gas belonging to a galaxy whose eventual merger with the massive elliptical host triggers the jets responsible for the RG. The gradual transfer of the angular momentum into the hot CGM of the host elliptical flattens its CGM into a fat-pancake shaped superdisk, which can account for the strip-like emission gaps observed between the radio lobes of such RGs. In this picture, the grossly off-centered location of the host (relative to the central plane of the SD) is relatively straightforward to understand in terms of the motion of the host galaxy during the period of jet activity, partly assisted by a linear momentum kick imparted by the merged galaxy. A corollary to this picture is that the SD material (which appears to be "docking the tails" at least in these RGs (cf., Jenkins \& Scheuer, 1976) is not gravitationally bound to the radio-loud elliptical. Interestingly, this scenario also provides a possible physical link between the SDs and the XRGs that are widely believed to manifest mergers of two galaxies containing SMBHs. 
The merger is likely to launch a pair of jets and if the host had already been recently active, a double-double radio structure can arise, as seen in three RGs in the present sample $(0035+130,0114-476$, and $1155+256)$. It is interesting that for between 10 and 13 of these 16 RGs, the brighter of the two outer hotspots is associated with the radio lobe adjoining the host galaxy. This indicates a diminishing of a jet's radio emitting potential following a long passage through the denser thermal material associated with the SD, as compared to a jet that mainly traverses the (light) relativistic plasma within a radio lobe. Perhaps a striking manifestation of this is seen in the RGs $0106+729$ and $1939+605$ where the radio jet appears to turn dissipative after propagating through the SD. No such dissipation is observed, at least in the present sample, for the jets that propagate mainly through the radio lobe. This calls for a follow-up study once a significantly larger sample of highly asymmetric superdisks becomes available.

We intend to investigate the realm of applicability of this model through simulations of the merger of two massive ellipticals with substantial circumgalactic gas.

\section{Acknowledgments}

We thank Santosh Joshi for assistance in compiling the present sample, Scott Tremaine for correspondence and Drs. Baum, Leahy and Owen for permission to republish maps. We appreciate the careful comments of the anonymous referee that have led to a significant improvement in the presentation of these results. This research has made use of the NASA/IPAC Extragalactic Database (NED) which is operated by the Jet Propulsion Laboratory, under contract with NASA. PJW is grateful for hospitality at NCRA, Princeton University and the Institute for Advanced Study.

\section{References}

Barai, P., Wiita, P.J., 2007. Testing Models of Radio Galaxy Evolution and the Cosmological Impact of FR II Radio Galaxies, ApJ, 658, 217-231.

Barthel, P.D., 1989. Is every quasar beamed? ApJ, 336, 606-611.

Baum, S.A., Heckman, T.M., Bridle, A., van Breugel, W.J.M., Miley G. K., 1988. Extended optical-line-emitting gas in radio galaxies - Broad-band optical, narrow-band optical, and radio imaging of a representative sample, ApJS, 68, 643-714.

Becker, R.H., White, R.L., Helfand, D.J., 1995. The FIRST Survey: Faint Images of the Radio Sky at Twenty Centimeters, ApJ, 450, 559-577. 
Bertin, G., Liseikina, T., Pegoraro, F., 2003. Slow evolution of elliptical galaxies induced by dynamical friction. I. Capture of a system of satellites, A\&A, 405, 73-88.

Black, A.R.S., Baum, S.A., Leahy, J.P., Perley, R.A., Riley, J.M., Scheuer, P.A.G., 1992. A study of FRII radio galaxies with $\mathrm{z}$ less than 0.15. I High-resolution maps of eight sources at $3.6 \mathrm{~cm}$, MNRAS, 256, 186-208.

Bogers, W.J., Hes, R., Barthel, P.D., Zensus, J.A., 1994. High resolution radio observations of intermediate redshift quasars and radio galaxies, A\&AS, 105, 91-113.

Burns, J.O., Basart, J.P., De Young, D.S., Ghiglia, D.C., 1984. Radio jets in classical double radio sources with strong cores, ApJ, 283, 515-528

Campbell-Wilson, D., Hunstead, R.W., 1994. Variable radio sources at 843 $\mathrm{MHz}, \mathrm{PASA}, 11,33-38$

Capetti, A., Morganti, R., Parma, P., Fanti, R., 1993. Polarization in low luminosity radio galaxies, A\&AS, 99, 407-435

Carilli, C. L., Wang, R., 2006. CO Line Width Differences in Early Universe Molecular Emission-Line Galaxies: Submillimeter Galaxies versus QSO Hosts, AJ, 131, 2763-2765

Cheung, C. C., 2007. FIRST "Winged" and X-Shaped Radio Source Candidates, AJ, 133, 2097-2121.

Croston, J.H., Birkinshaw, M., Hardcastle, M.J., Worrall, D.M., 2004. X-ray emission from the nuclei, lobes and hot-gas environments of two FR II radio galaxies, MNRAS, 353, 879-889.

Di Matteo, P., Combes, F., Melchior, A.-L., Semelin, B., 2007. Star formation efficiency in galaxy interactions and mergers: a statistical study, A\&A, 468, 61

Ferrara, A., Perna, R., 2001. Scintillation as a probe of the intergalactic medium, MNRAS, 325, 1643-1648.

Garrington, S.T., Leahy, J.P., Conway, R.G., Laing, R.A., 1988. A systematic asymmetry in the polarization properties of double radio sources with one jet, Nature, 331, 147-149.

Garrington, S.T., Conway, R. G., Leahy, J. P., 1991. Asymmetric depolarization in double radio sources with one-sided jets, MNRAS, 250, 171-197.

Gergely, L.., Biermann, P.L., 2007. Supermassive black hole binary mergers, preprint [arXiv0704.1968]

Gilbert, G.M., Riley, J.M., Hardcastle, M.J., Croston, J.H., Pooley, G.G., Alexander, P., 2004. High-resolution observations of a complete sample of $27 \mathrm{FR}$ II radio galaxies and quasars with $0.3<z<0.6$, MNRAS, 351, 845-890.

González-García, A.C., van Albada, T.S., 2005. Encounters between spherical galaxies - I. Systems without a dark halo, MNRAS, 361, 1030-1042.

Gopal-Krishna, 1991. An Intergalactic Origin for the Low Frequency Flux Variations of Extragalactic Radio Sources, Current Sci., 60, 117-122.

Gopal-Krishna, Biermann, P.L., Wiita, P.J., 2003. The Origin of X-shaped Radio Galaxies: Clues from the Z-symmetric Secondary Lobes, ApJ, 594, 


\section{L103-L106 (GKBW03).}

Gopal-Krishna, Nath, B.B., 1997. Accretion of gaseous disks at high redshifts and the depolarization asymmetry of radio-loud quasars, A\&A, 326, 45-50.

Gopal-Krishna, Wiita, P.J., 1996. On the Origin of Correlated Radio-optical Asymmetries in Double Radio Sources, ApJ, 467, 191-196.

Gopal-Krishna, Wiita, P.J., 2000. Superdisks in Radio Galaxies, ApJ, 529, 189-200 (GKW00).

Gopal-Krishna, Wiita, P.J., 2005. Asymmetries in Powerful Extragalactic Radio Sources, in Saha S.K., Rastogi V.K., (Eds.) 21st Century Astrophysics, Anita Publications, New Delhi, p. 108-133 (astro-ph/0409761).

Gopal-Krishna, Wiita, P.J., Joshi, S., 2007. Superdiscs in radio galaxies: jetwind interactions, MNRAS, 380, 703-711.

Hardcastle, M.J., Alexander, P., Pooley, G.G., Riley, J.M., 1997. Highresolution observations at $3.6 \mathrm{~cm}$ of seventeen FRII radio galaxies with $0.15<z<0.30$, MNRAS, 288, 859-890.

Harvanek, M., Hardcastle, M.J., 1998, A VLA Study of 15 3CR Radio Galaxies, ApJS, 119, 25-39.

Heinz, S., Brüggen, M., Young, A., Levesque, E., 2006. The answer is blowing in the wind: simulating the interaction of jets with dynamic cluster atmospheres, MNRAS, 373, L65-L69.

Humphrey, A., Villar-Martin, M., Fosbury, R., Binette, L., Vernet, J., De Brueck, C., di Serego Aligheiri, S., 2007. Giant Ly $\alpha$ nebulae around $z>2$ radio galaxies: evidence for infall, MNRAS, 375, 705-714

Jahnke, K., Wisotzki, L., 2003. The B-band luminosities of quasar host galaxies, MNRAS, 346, 304-318.

Jeltema, T.E., Mulchaey, J.S., Lubin, L.M., Rosati, P., Böhringer, H., 2006. X-Ray Properties of Intermediate-Redshift Groups of Galaxies, ApJ, 649, 649-660.

Jenkins, C. J., Scheuer, P. A. G., 1976. What docks the tails of radio source components?, MNRAS, 174, 327-333

Jones, T.W., De Young, D.S., 2005. Magnetohydrodynamic Simulations of Relic Radio Bubbles in Clusters, ApJ, 624, 586-605.

Laing, R.A., 1988. The sidedness of jets and depolarization in powerful extragalactic radio sources, Nature, 331, 149-151.

Lara, L., Márquez, I., Cotton, W.D., Feretti, L., Giovannini, G., Marcaide, J.M., Venturi, T., 1999. Restarting activity in the giant radio galaxy J1835+620, A\&A, 348, 699-704.

Lara, L., Cotton, W.D., Feretti, L., Giovannini, G., Marcaide, J.M., Márquez, I., Venturi, T., 2001. A new sample of large angular size radio galaxies. I. The radio data, A\&A, 370, 409-425.

Leahy, J.P., Perley, R.A., 1991. VLA images of 23 extragalactic radio sources, AJ, 102, 537-561.

Leahy, J.P., Williams, A.G., 1984. The bridges of classical double radio sources, MNRAS, 210, 929-951.

Leahy, J.P., Black, A.R.S., Dennett-Thorpe, J., Hardcastle, M.J., Komissarov, 
S., Perley, R.A., Riley, J.M., Scheuer, P.A.G., 1997. A study of FRII radio galaxies with $z<0.15$ - II. High-resolution maps of 11 sources at $3.6 \mathrm{~cm}$, MNRAS, 291, 20-53.

Mathews, W.G., Brighenti, F., 2003. Hot Gas in and around Elliptical Galaxies, ARA\&A, 41, 191-239.

McCarthy, P.J., van Breugel, W., Kapahi, V.K., 1991. Correlated radio and optical asymmetries in powerful radio sources, ApJ, 371, 478-490.

Merritt, D., Ekers, R.D., 2002. Tracing Black Hole Mergers Through Radio Lobe Morphology, Science, 297, 1310-1313.

Morganti, R., Killeen, N.E.B., Tadhunter, C.N., 1993. The Radio Structures of Southern 2-Jy Radio Sources, MNRAS, 263, 1023-1048. .

Morganti, R., Oosterloo, T., Tadhunter, C.N., Aiudi, R., Jones, P., VillarMartin, M., 1999. The radio structures of southern 2-Jy radio sources: New ATCA and VLA radio images, A\&AS, 140, 355-372.

Mori, M., Burkert, A., 2000. Gas Stripping of Dwarf Galaxies in Clusters of Galaxies, ApJ, 538, 559-568.

Narayanan, D. et al., 2008. The Nature of CO Emission from z 6 Quasars, ApJS, 174, 13-30.

Noel-Storr, J., Baum, S.A., Verdoes Kleijn, G., van der Marel, R.P., O’Dea, C.P., de Zeeuw, P.T., Carollo, C.M., 2003. Space Telescope Imaging Spectrograph Spectroscopy of the Emission-Line Gas in the Nuclei of Nearby FR-I Galaxies, ApJS, 148, 419-472.

Owen, F.N., Ledlow, M.J., 1997. A 20 Centimeter VLA Survey of Abell Clusters of Galaxies. VII. Detailed Radio Images, ApJS, 108, 41-98.

Parkinson, H., Cole, S., \& Helly, J., 2008. Generating dark matter halo merger trees, MNRAS, 383, 557-564.

Perley, R. A., Taylor, G. B. 1991, VLA observations of 3C 295 - A young radio galaxy? AJ, 101, 1623-1631.

Roediger, E., Hensler, G., 2005. Ram pressure stripping of disk galaxies. From high to low density environments, A\&A, 433, 875-895.

Rottmann, H., 2001. PhD thesis, Univ. Bonn

Saripalli, L., Subrahmanyan, R., \& Udaya Shankar, N. 2002, A Case for Renewed Activity in the Giant Radio Galaxy J0116-473, ApJ, 565, 256-264.

Scheuer, P.A.G., 1974. Models of extragalactic radio sources with a continuous energy supply from a central object, MNRAS, 166, 513-528.

Schoenmakers, A.P., de Bruyn, A.G., Röttgering, H.J.A., van der Laan, H., Kaiser, C.R., 2000. Radio galaxies with a 'double-double morphology' - I. Analysis of the radio properties and evidence for interrupted activity in active galactic nuclei, MNRAS, 315, 371-380.

Soker, N., Pizzolato, F., 2005. Feedback Heating with Slow Jets in Cooling Flow Clusters, ApJ, 622, 847-852.

Statler, T.S., McNamara, B.R., 2002. A 15 Kiloparsec X-Ray Disk in the Elliptical Galaxy NGC 1700, ApJ, 581, 1032-1038.

Tadhunter, C. et al., 2007. The Heating Mechanism for the Warm/Cool Dust in Powerful, Radio-loud, Active Galactic Nuclei, ApJ, 661, L13-L17 
van Breugel, W., Jagers, W., 1982. Multifrequency observations of extended radio galaxies $\mathrm{V}-3 \mathrm{C} 31,3 \mathrm{C} 33.1,3 \mathrm{C} 35,3 \mathrm{C} 66 \mathrm{~B}, 3 \mathrm{C} 129,3 \mathrm{C} 130,3 \mathrm{C} 223$, 3C 310, 3C 390.3 and 4C 48.29, A\&AS, 49, 529-559.

Wiita, P.J., Gopal-Krishna, 1990. On spectral aging in lobes of double radio sources, ApJ, 353, 476-479.

Wiita, P.J., Norman, M.L.., 1992. Numerical simulations of hydrodynamical jets crossing a galactic halo/intracluster medium interface, ApJ, 385, 478490.

Wilson, A.S., Colbert, E.J.M., 1995. The difference between radio-loud and radio-quiet active galaxies, ApJ, 438, 62-71.

Wold, M., Lacy, M., Lilje, P.B., Serjeant, S., 2000. Clustering of galaxies around radio quasars at $0.5 \leq z \leq 0.8$, MNRAS, 316, 267-282.

Zier, C., 2005. Orientation and size of the ' $\mathrm{Z}$ ' in $\mathrm{X}$-shaped radio galaxies, MNRAS, 364, 583-592.

Zier, C., Biermann, P.L., 2002. Binary black holes and tori in AGN. I. Ejection of stars and merging of the binary, A\&A, 377, 23-43. 